\title{
Anna Maria RAO (sous la direction de), Foules contre-révolutionnaires. Les insurrections populaires dans l'Italie jacobine et napoléonienne
}

\section{Carlo Capra}

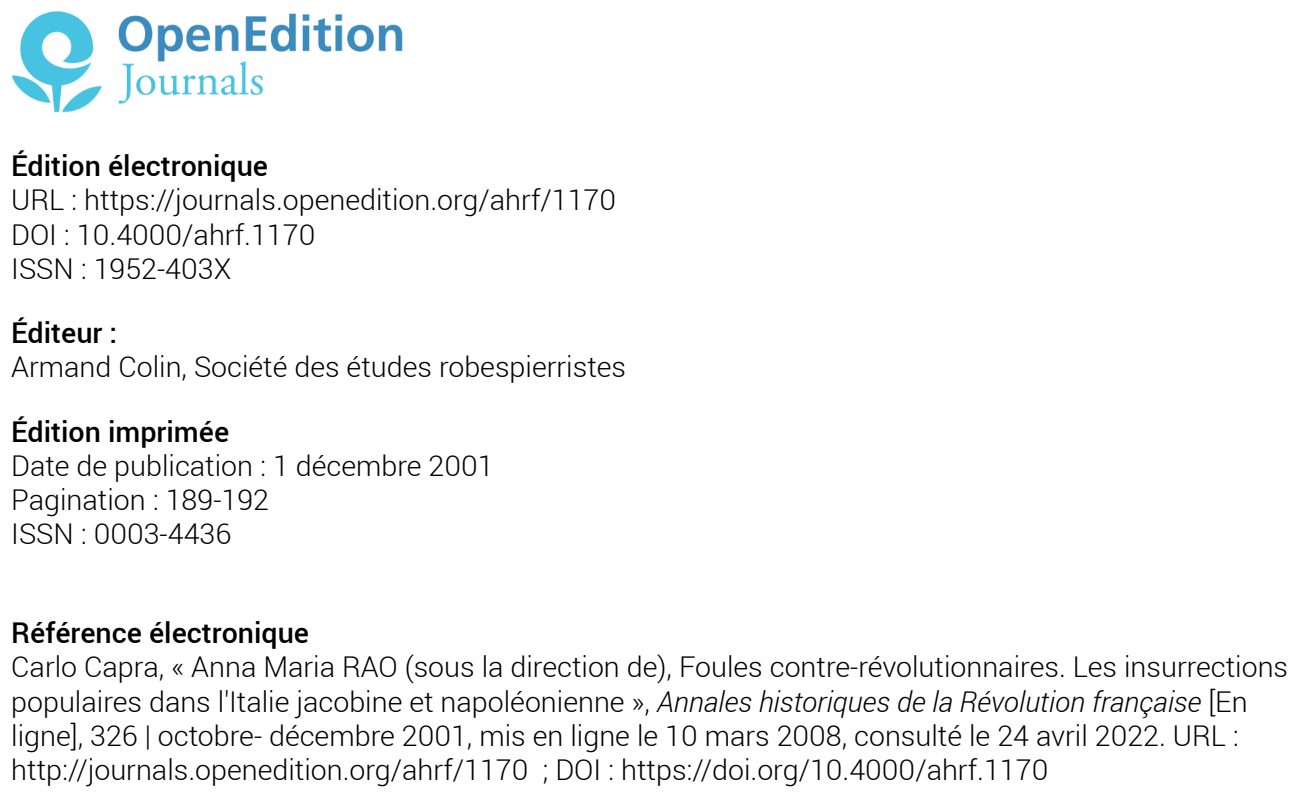

Ce document a été généré automatiquement le 24 avril 2022.

Tous droits réservés 


\title{
Anna Maria RAO (sous la direction de), Foules contre-révolutionnaires. Les insurrections populaires dans l'Italie jacobine et napoléonienne
}

\author{
Carlo Capra
}

\section{NOTE DE L'ÉDITEUR}

Traduction B. Gainot

1 Les célébrations du bicentenaire du «triennio républicain » 1796-1799 (la dénomination apparaît nettement préférable à celle de «triennio jacobin " pour les raisons justement exposées par Luciano Guerci) n'ont pu faire autrement qu'enregistrer le changement de climat politico-culturel, et qu'être marquées par le courant révisionniste, dont les premiers effets s'étaient manifestés à l'occasion du bicentenaire de la Révolution française. L'une des voies principales prises par cette polémique contre les orientations historiographiques dominantes fut l'accent mis sur les mouvements sanfé-distes et contre-révolutionnaires qui se produisirent au cours de ces années dans pratiquement toutes les régions italiennes, interprétés et exaltés dès lors comme l'expression d'un sentiment national viscéral et d'une réaction contre les étrangers venus fouler aux pieds notre terre (mais n'y eut-il pas à maintes reprises des étrangers dominateurs et spoliateurs, pourrions-nous faire remarquer d'emblée ?), interprétés tantôt comme la rencontre de mœurs ancestrales et de la religion traditionnelle, outragées par les Français impies et leurs alliés, tantôt comme la défense des petites patries locales et de leur autonomie. Il ne serait pas difficile de rattacher chacune de ces interprétations, qui se présentent souvent sous un aspect confus, à tel ou tel courant politico idéologique. Aucun scandale, cela va de soi, sauf à remarquer que l'idéologie n'est pas uniquement le fait de la gauche, et que le seul résultat qui compte est la solidité de la recherche et l'originalité des conclusions auxquelles on aboutit. De ce point de vue, toutefois, il ne 
semble pas que la prolifération des publications, des revues, des colloques, consacrés aux insurrections, ouvre des perspectives d'une grande nouveauté, étant donnée la primauté, par rapport aux études précises et à la publication documentaire, des affirmations de principe, reprises pour la plupart des travaux anciens de Lumbroso et Rodolico (réédité récemment pour la premier avec une préface de Oscar Sanguinetti), et des polémiques contre l'historiographie "officielle", accusée de négliger et de sousestimer les mouvements contre-révolutionnaires. Sur le bien-fondé de ces accusations, il y aurait matière à discussion, si on a en mémoire des études aussi importantes que celles de Cingari et Turi dans les années cinquante et soixante, et plus récemment les travaux de Rao et Davis sur le Mezzogiorno, de Minciotti Tsoukas et Cattaneo sur les États de l'Église, de Ricuperati et Broers sur le Piémont, pour m'en tenir aux premières références qui me viennent à l'esprit. À celles-ci, on peut ajouter les articles réunis dans le numéro spécial de Studi Storici d'avril-juin 1998, rassemblés par Anna Maria Rao, et intitulés: Les insurrections contre-révolutionnaires dans l'Italie révolutionnaire et napoléonienne, il est particulièrement utile de les trouver désormais réunis en un seul volume, moyennant quelques retouches, et un supplément de deux articles, l'un de Mattone et Sanna sur les mouvements populaires sardes entre 1793 et 1796, l'autre de Francesca Maria Lo Faro sur la révolution et la contre-révolution dans la région de Bari.

De l'ensemble de ces études, l'insurrection ressort - selon Anna Maria Rao -, dans toute son inexorable complexité, comme un phénomène diversifié dans l'espace et dans le temps, faisant ressortir une multiplicité de situations locales, une variabilité extrême de la dynamique et des objectifs [...] qui rendent difficile toute tentative de généralisation abstraite (p. 12). Ce n'est pas partout, avant tout, que les mouvements populaires prennent une direction clairement anti-française et anti-révolutionnaire. La meilleure preuve du caractère ambigu, ou plus exactement pré-politique, de la contestation, est fournie par les deux monarchies les plus anciennes et les plus fortement enracinées dans la tradition, le Royaume de Sardaigne et le Royaume de Naples. Pour le premier, il faut distinguer nettement les conditions de l'île de Sardaigne, où c'est dans une période récente que s'est fait sentir la politique centralisatrice de la Maison de Savoie, et les conditions des communautés piémontaises, habituées depuis des siècles à la présence et au pouvoir d'arbitrage des fonctionnaires royaux. La "révolution sarde » qui s'est traduite par l'élaboration d'un programme "constitutionnel», oeuvre des Stamenti en 1793, par l'expulsion des Piémontais, puis par le développement d'un mouvement anti-féodal de grande ampleur, est certainement un cas unique, qui ressemble beaucoup plus aux événements contemporains de la Corse qu'aux événements qui se passent dans la péninsule, et représentent en même temps un contrepoint à la tendance à tout vouloir ramener en termes d'adhésion ou de réaction aux idées françaises, et une réaffirmation de la nécessité de replacer les faits du Triennio dans une perspective à plus long terme. Les troubles qui éclatent dans une quarantaine de communautés du Piémont méridional au mois de juillet 1797, tels que les reconstitue Alice Blythe Raviola à partir d'une large documentation inédite, ne peuvent se comprendre sans tenir compte de la grave crise économique et sociale qui secoue la région depuis le début de la décennie 1790, et en même temps, de l'affaiblissement du contrôle des autorités en raison de la défaite militaire. Reste le fait que, à la base du mouvement, auquel prennent part le peuple et les bourgeois, il n'y a pas seulement la protestation contre la vie chère, mais aussi un sentiment égalitaire qui s'exprime par la lutte contre les droits féodaux, et par des revendications parfois naïves, comme la requête avancée par les habitants de Racconigi selon laquelle les messieurs de la paroisse ne devront commettre aucune injustice en sonnant 
les cloches, à l'occasion des funérailles, et devront sonner les cloches aussi bien pour les pauvres que pour les riches (p. 131) ; on trouve ici une certaine ressemblance avec la Grande Peur analysée par Lefebvre. De même, à l'autre extrémité de la péninsule, dans la région de Bari, la haine de l'étranger et le sentiment religieux ne paraissent pas avoir joué un rôle significatif. Souvent, dans les cités populeuses comme dans les petits centres urbains, les troubles étaient une manifestation des enjeux locaux de pouvoir (p. 327) avec un arrière-plan de crise économique et de subsistances, comme l'attestent en 1799 les manifestations d'adhésion à la plantation des arbres de la liberté, et autres cérémonies républicaines. Le triomphe final des forces légitimistes apparaît plutôt comme une conséquence de l'évolution de la situation générale du Royaume (la marche victorieuse du cardinal Ruffo), et non comme un choix spontané des populations. Il est avéré que les sympathies républicaines caractérisent plusieurs petits territoires dans le Basilicate voisin (non traité dans les contributions); Anna Maria Rao observe avec pertinence dans l'introduction que la participation populaire au mouvement républicain [...] est généralement sous-estimée et nécessiterait une prise en considération (p.23). Plus généralement, elle semble partager le point de vue exprimé dans l'article de John A. Davis (Révoltes populaires et contre-révolution dans le Mezzogiorno continental) sur la base d'études récentes qui montrent que les insurrections populaires furent motivées par des conflits qui prenaient racine dans la lente transformation du régime féodal du Mezzogiorno, prenant la forme d'une guerre persistante entre les riches et les pauvres, prompte à éclater à la première occasion de crise politique (p. 350).

3 Ce qui ne veut pas dire, naturellement, que toutes ces remarques conduisent à négliger la dimension anthropologique du phénomène, qui présente des aspects rituels et mentaux tout aussi révélateurs de la profondeur de la crise qui a secoué la société, non seulement italienne, mais également européenne, à la fin du siècle (Rao, p. 29). Pour cette dimension, il faut pleinement prendre en compte le sentiment religieux populaire qui, particulièrement en Italie centrale, fut largement dominant, et mobilisé pour servir la propagande antifrançaise, insistante et véhiculée par le clergé à partir de 1791, comme le montre la vague de «miracles» qui parcourt ces régions au début de l'invasion française. Trois articles sont consacrés aux États de l'Eglise dans ce volume. Massimo Cattaneo, auteur en 1995 d'une importante étude (Le regard de Marie sur la Révolution) sur les miracles de 1796-1797, s'intéresse ici à l'opposition populaire au jacobinisme à Rome et dans les États de l'Église, s'appuyant en particulier sur la révolte trastevérine du 25 février 1798. Marina Caffiero, dans l'unique article du volume qui soit consacré aux premières années du $\mathrm{XIX}^{\mathrm{e}}$ siècle, analyse les traits de l'action répressive (en fait bien légère et modérée) menée par le gouvernement pontifical restauré contre les «jacobins»: ce qui lui permet d'exploiter une très intéressante source judiciaire relative à une nouvelle vague d'insurrections en 1806, et d'affirmer que leurs motivations tendent à souligner une compétition désormais installée entre deux factions locales ou "partis" pour lesquels, derrière les formes "archaïques» de la rivalité pour le pouvoir, se mêlent d'anciennes rancœurs, des inimitiés personnelles, des rivalités de familles et de clans, des désirs de vengeance et des conflits de groupes, internes à la communauté, et l'esprit de clocher, entre communautés voisines traditionnellement rivales (p.317). Ces observations mériteraient d'être étendues à d'autres espaces de la péninsule, où les conflits du Triennio ont laissé un reliquat de haine, et ont contribué à la cristallisation d'antagonismes ancrés dès lors dans la longue durée (selon le modèle départemental sarthois étudié par Paul Bois). C'est un autre champ de recherches qui peut ainsi s'ouvrir, plus généralement, autour de la crainte de la violence populaire engendrée par les événements de 1796-1799, avec sa trace dans la 
mentalité des propriétaires, et dans les attitudes des intellectuels, selon des exemples bien connus, tels que ceux de Cuoco ou de Foscolo. Les insurrections anti-françaises de la région de Ferrare sont enfin l'objet d'une ample contribution de Valentino Sani, qui repère une double origine de la révolte de Lugo et Cento, dans l'été 1796 ; d'un côté, une authentique croisade pour la défense de la Sainte Foi et du pouvoir temporel des papes, qui traduit les sentiments du petit peuple, de l'autre, une stratégie politique des notables soulevés pour l'affirmation d'une autonomie pleine et entière du Ferrarais (pp. 199-200).

Sur la périphérie des États de l'Église, nous assistons également à cette renaissance de l'esprit citadin et municipal, qui avait déjà retenu l'attention de Franco Venturi, et qui constitue le tournant paradoxal de l'ébauche d'un projet d'unification nationale ; à la démocratisation du chef-lieu, pour laquelle entre pour une part essentielle la volonté d'émancipation par rapport à la sujétion pontificale, répond le choix contraire des bourgeois les plus nantis. Ce processus, mutatis mutandis, est à l'œuvre dans la révolte des vallées de Brescia et de Bergame, pour laquelle par ailleurs le sentiment religieux et le rejet des Français occupent une place beaucoup moins importante que dans le Ferrarais, comme le montre l'article de Paolo Preto. Plus complexe est le cas des "Pâques véronaises", qui mêle citadins et ruraux; parmi ses multiples causes, Gian Paolo Romagnani énumère la tension entretenue depuis dix mois par l'occupation militaire, le poids des réquisitions sur une économie déjà affaiblie par la crise des années 90 , le traditionnel attachement des paysans et du petit peuple à l'église et à la paroisse, le rôle décisif d'une noblesse citadine qui possède des attaches terriennes et une multitude de réseaux d'alliance qui ont tenu enserrée jusqu'à cette époque la société locale dans un équilibre fragile mais bien réel entre la ville et la campagne (p. 117).

La situation de la Ligurie relève encore d'une autre configuration: une révolte antijacobine et " patriotique » du petit peuple de Gênes, étudiée par Giovanni Assereto, une sorte de réplique de la révolte populaire de 1746 contre les Autrichiens (p.173), voit coïncider en mai 1796, sans se confondre pour autant, un large soulèvement des paysans des vallées, encadré par le clergé et les autorités locales, qui sera suivi de la brève mais cruelle insurrection des Viva Maria au début de septembre. Le mouvement s'unifie autour de la défense de la religion qu'on jugeait mise en péril par la constitution démocratique; mais cet élément n'en cache pas moins au regard acéré d'Assereto la variété des revendications locales : Même si elle prit la forme d'une jacquerie, l'insurrection fut diverse : elle dessine sur la carte de la région ligurienne, avec une remarquable précision, la carte de la fidélité à l'Ancien Régime et à ses valeurs. Une fidélité qui prend des formes différentes selon les localités: à la périphérie de la capitale, là où domine le système agricole du grand domaine, elle naît de la condition privilégiée de ceux qui vivent sur les tenures nobiliaires en quasi-symbiose avec les propriétaires aristocrates; dans les Monts ligures, elle hérite d'une ancienne dévotion de nature féodale, et de l'orgueil d'appartenir à de petites communautés dotées par l'État de lois et de coutumes particulières; tandis qu'à Fontanabuona et autres vallées du Levante, nous nous trouvons face à une organisation politico-sociale archaïque qui avait de tout temps trouvé son équilibre propre dans le cadre de l'état génois, sans pour autant s'identifer à lui de façon permanente (pp. 189-190).

La participation au mouvement d'éléments de la classe dominante, avec leurs fonctions organisatrices et dominatrices, a été davantage suggérée que précisément démontrée par les études précédentes. C'est ce qui fait l'intérêt de l'article de Claudio Tosi qui, partant de l'analyse d'archives privées, reconstitue le portrait social et culturel d'un protagoniste de premier plan de l'insurrection aretine de 1799, le marquis colonel 
Giovan Battista Albergotti; un réactionnaire, certes, mais d'une toute autre nature que celle d'un fanatique mû exclusivement par l'intolérance religieuse ou le légitimisme aveugle; plutôt un noble rusé et sans préjugés, doté d'une notable capacité d'organisation, rattaché au courant majoritaire de l'aristocratie européenne qui, au moins dans une première phase, saisit l'occasion de la poussée contre-révolutionnaire pour chercher à reconstruire les conditions de l'Ancien Régime contre l'œuvre réformatrice des souverains absolus (p. 252). La contribution de Tosi apporte ainsi une illustration utile - mais ce n'est pas une critique - à la thèse de Gabriel Turi, récemment rééditée avec une vaste postface (Viva Maria! Réforme, révolution et insurrection en Toscane, Bologne, II, Mulino, 1999) selon laquelle l'insurrection de 1799 est l'acte final d'une protestation populaire contre les répercussions négatives des réformes léopoldines sur le plan économique et social. Contre toute généralisation hâtive, contre toute lecture désinvolte, de nature apologétique et idéologique, en vertu de laquelle le sanfedisme, plutôt qu'un objet d'étude, apparaît comme un projet anachronique pour le présent (selon M. Cattaneo, Contrerévolutions et insurrections in D. Amando - M. Cattaneo - M. P. Donato : Une révolution difficile. La république romaine de 1798-1799, Pisa-Roma, Istituto editoriali e poligrafici internazionali, 2000, p. 183), il est nécessaire de réaffirmer l'exigence (non négociable) de la seule recherche qui soit à la fois appuyée sur des documents et étayée sur le plan méthodologique, à l'image de celles qui composent le présent volume, rattachant les événements des années 1796-1799 à l'époque qui les précède, sachant relier aux grandes évolutions nationales et internationales de la période - par exemple, les effets de la propagande contre-révolutionnaire de l'Église ou la dégradation des conditions de vie du peuple - aux tensions et aux conflits engendrés par les circonstances de la crise et les rapports de forces locaux. 